\title{
La Escuela Bosque como modelo de escuela alternativa: antecedentes, características y repercusión
}

\author{
Ana Pérez de Ontiveros Molina \\ Facultad de Educación. Universidad de Murcia, España. anaperezontiveros@gmail.com \\ ORCID: https://orcid.org/0000-0001-6148-8626
}

[Recibido: 15 diciembre 2020. Revisado: 10 mayo 2021. Aceptado: 14 junio 2021]

\begin{abstract}
Resumen: Este estudio tiene como objetivo principal dar a conocer el funcionamiento de las Escuelas Bosque, que forman parte de un tipo de educación conocida como educación alternativa. Para ello, se plasmarán los acontecimientos históricos que dieron lugar a su origen y su posterior desarrollo. Más adelante, se expondrán definiciones que han intentado explicar este tipo de modelo educativo y sus principales características. Tras esto, se hará referencia a las repercusiones de este tipo de escuela, sus ventajas e inconvenientes. Por último, se esbozará la situación de este modelo educativo en la pandemia.
\end{abstract}

Esto se ha llevado a cabo a través de una indagación teórica y análisis bibliográfico, recogiendo los datos científicos más relevantes sobre el tema. Por tanto, los resultados que se han obtenido reflejan un modelo educativo que está implantado en Europa y que está emergiendo progresivamente en España.

Palabras clave: educación infantil; educación en la naturaleza; escuelas alternativas; metodología activa; pandemia.

\section{Forest School as a model of an alternative school}

Abstract: The main objective of this research is to provide information about the functioning of Forest Schools, which are part of an education group known as alternative education. With this aim, the historical events that gave rise to its origin and subsequent development will be reflected. Later, definitions that have tried to explain this type of educational model and its main characteristics will be presented. After this, reference will be made to the repercussions of this type of school, advantages and disadvantages. Finally, the situation of this educational model in pandemic will be outlined.

This study has been carried out through a theoretical investigation and bibliographic analysis, collecting the most relevant scientific data on the subject. Therefore, the results that have been obtained reflect an educational model that is implanted in Europe and that is emerging progressively in Spain.

Keywords: early childhood education; education in nature; alternative schools; active methodology; pandemic.

Para citar este artículo: Pérez de Ontiveros Molina, A. (2021) La Escuela Bosque como modelo de escuela alternativa: antecedentes, características y repercusión. Revista de Educación Ambiental y Sostenibilidad 3(1), 1303. doi: 10.25267/Rev_educ_ambient_sostenibilidad.2021.v3.i1.1303

\section{Introducción}

La creciente urbanización y desforestación de las áreas rurales trae consigo una desnaturalización de las personas y muchos efectos sobre la naturaleza. Según el informe Planeta Vivo de 2018 (Grooten y Almond, 2018, p. 145), "los principales factores responsables de la pérdida de diversidad son la sobrexplotación y la agricultura, y ambos son el resultado del crecimiento ininterrumpido del consumo humano" 
Como consecuencia de este comportamiento se ha producido la deforestación de las zonas rurales, la explotación de los recursos medioambientales, la contaminación de mares y ríos, etc.

Por su parte, Sapiains (2010, citado en Acuña, Díaz y Ramírez, 2018) habla de una cosificación del medio natural, que pasa a ser una fuente inagotable de recursos. Esto significa que se produce un continuo abandono de lo natural en beneficio del abuso de las nuevas tecnologías o de la colonización y urbanización de los espacios naturales.

A su vez, es muy extraño que los niños de hoy prefieran, en la mayoría de los casos, una tarde en la que han conseguido el permiso de sus padres para jugar con la tableta durante más de una hora, en lugar de divertirse realizando una tarea tan satisfactoria como la de construir una cabaña en el monte durante horas. Según Elkind (2007, citado en Freire, 2011), el tiempo que un niño pasa jugando a la semana puede llegar a ser de tan solo 12 horas. Asimismo, según Gil (2007, citado en Peris, 2017), los niños de hoy tienen cada vez más actitudes propias de los adultos, ya que desde hace 30 años los adultos han comenzado a reducir la libertad de los niños y tener mucho más control del que tenían antiguamente.

Como consecuencia de esta situación, muchas escuelas se animan a incorporar la naturaleza como espacio de aprendizaje. Algunas las utilizan como aula alternativa a los espacios cerrados con los que cuentan. Este es el caso (entre muchos) del Colegio público Félix Rodríguez de la Fuente de Murcia, que, debido a la actual situación pandémica, decide salir a la playa cada mañana a dar sus clases (Ribelles, 2021). Por otro lado, muchos otros colegios utilizan el medio natural como espacio y recurso de aprendizaje. Estas escuelas son llamadas Escuelas Bosque, Escuelas en la Naturaleza, Bosquescuela, Playa Escuela, etc.

Este artículo se centrará en realizar una breve síntesis de las principales características de las Escuelas Bosque, sus orígenes y sus beneficios e inconvenientes. Esto se hará para dar a conocer este modelo de educación que cada vez cobra más importancia en nuestro país y, por otro, llevar la esencia de estas escuelas a la educación convencional. Cabe destacar que se hablará de ellas como una de las principales metodologías activas.

\section{Antecedentes}

Los movimientos pedagógicos actuales que sostienen la relevancia de la conexión del niño con la naturaleza tienen su raíz en las ideas de muchos autores como Rousseau, Locke, Schelling, Basedow, Pestalozzi y Fröebel (Yildirim y Özyilmaz, 2017). Este último era uno de los defensores de la creación de modelos educativos que tuvieran en cuenta el contacto de los niños con la naturaleza, el cual propone "grandes salas en las que el aire se mantenga puro para un número limitado de alumnos que puedan moverse con libertad, espacios semicubiertos y campos extensos, donde se renueve el aire de la infancia (...)" (Lahoz, 1991, p.124).

A finales del siglo XIX, en 1876, se creó en España la Institución Libre de Enseñanza (en adelante, ILE), como respuesta a la expulsión de numerosos catedráticos de la universidad que, influenciados por la corriente krausista, defendían que la universidad no debía regirse por dogmas religiosos, sino que debía fomentar el conocimiento científico y dar libertad de pensamiento tanto a alumnos como a 
profesores (Sanchidrián y Ruiz, 2010). Fundada por Giner de los Ríos, esta institución se constituyó como una de las escuelas más innovadoras de Europa.

Algunas de las aportaciones más importantes de Francisco Giner fueron, precisamente, las excursiones escolares y la defensa del juego al aire libre. Según él, los niños debían aprender las cosas desde su lugar natural, el cual, obviamente, estaba fuera del aula.

Siguiendo estos nuevos pensamientos, emergió un movimiento durante los últimos años del siglo XIX, alrededor de 1875: la Escuela Nueva. Consistía en un movimiento heterogéneo que surgió a través de unas ideas sobre educación que iban en contra del modelo que estaba instaurado. Esto supuso romper con la educación tradicional basada en transmitir unos conocimientos fijos a través de la memorización y regios comportamientos, para pasar a un concepto completamente nuevo: libertad de elección del niño y valor hacia su individualidad y autonomía (Narváez, 2006).

En la actualidad existen numerosas escuelas que apuestan por la educación al aire libre y sitúan la naturaleza en la base de su proyecto educativo, pero, aunque sus propuestas parezcan novedosas, su origen se encuentra muchos años atrás.

La primera escuela al aire libre stricto sensu tiene lugar en Charlottenburg. En la primera década del siglo XX se llevó a cabo un movimiento promovido por médicos y clínicas que tenía como fin prevenir enfermedades como la tuberculosis, entre otras. Este movimiento iba dirigido a las familias de clase obrera que vivían en grandes ciudades $\mathrm{y}$, en muchas ocasiones, en situaciones precarias. Para abastecer las necesidades educativas de los niños, se creó la Waldschule. A partir de estas, surgieron otras experiencias como la Open Air School en Londres en 1908 o la Fresh Air School en Estados Unidos en 2011 (Bruchner, 2017a).

En España este concepto de escuela llegó a Barcelona de la mano de Rosa Sensat. Esta recibió en 1912 una beca de seis meses para realizar investigaciones científicas. La finalidad de su viaje era visitar una serie de escuelas primarias que llevaran la enseñanza de las ciencias y el estudio del medio a la base del desarrollo educativo (Aguado, 2018).

Tras su viaje, quedó deslumbrada por Decroly y su pedagogía, que tenía entre sus principios pedagógicos la observación de la naturaleza, los niños podían experimentar los objetos y aprender a través de los sentidos.

Dos años más tarde, Rosa Sensat funda, apoyada por el Ayuntamiento de Barcelona, l'Escola de Bosc de Montjuïc, la primera escuela al aire libre en España. En un principio, estaba destinada a alumnos con problemas de salud que necesitaban un espacio al aire libre como método curativo. Sin embargo, tras la llegada de Sensat, esta escuela se convirtió en un centro educativo ordinario, únicamente teniendo como aula el propio medio ambiente (Aguado, 2018).

Teniendo esta escuela como referente, se establece en Madrid en 1914 la Escuela Bosque de Dehesa de la Villa, la cual tuvo como primera directora a Flora Mateos de la Torre (Bruchner, 2017a).

Con los años, se fue perfeccionando esta forma de enseñar al aire libre y se llegó a lo que se conoce actualmente como Escuela Bosque. Esta nace en Dinamarca en los años cincuenta con el nombre de Udeskole (Colegio del bosque). Su proyección fue muy 
grande, llegando a muchos países europeos: Alemania, Finlandia, Noruega, Suecia... En países como Gran Bretaña con sus Forest Schools, e incluso países asiáticos como Corea del Sur, este tipo de proyecto educativo se lleva a cabo por el Ministerio de Educación y todos los niños tienen acceso a este tipo de colegios. Así también, en América Latina contamos con asociaciones como CIFREP (2021) que buscan dar visibilidad a un modelo educativo que tenga la naturaleza como protagonista.

En España el primer centro con estas características nació en 2011, como una respuesta a la necesidad de tres familias de encontrar un lugar tranquilo y en plena naturaleza, donde sus hijos pudieran comenzar a descubrir el mundo. El proyecto se denominó Grupo de Juego en la Naturaleza Saltamontes y actualmente está situado en la Sierra de Guadarrama. Este centro acoge a niños de tres a seis años y sus principios pedagógicos se basan en el respeto al niño y el juego espontáneo al aire libre (Hueso, 2012).

Tras ellos, se crea el primer centro homologado Escuela Bosque en España gracias a Philip Bruchner. Este es un educador alemán que ha promovido las pedagógicas al aire libre a lo largo de su trayectoria profesional y que ha querido fundar la Bosquescuela Cerceda de la mano de la empresa Interprende y la Fundación Félix Rodríguez de la Fuente. Esta última pretende que los alumnos, a través del contacto directo con la naturaleza, consigan aprender y desarrollarse plenamente como personas (Ruiz, 2017).

A raíz de esto, han surgido numerosos proyectos de estas características en los últimos años teniendo, actualmente, 16 en toda España (Ludus, 2021).

\section{Definición y características de las Escuelas Bosque}

Según Forminaya (2015, citado en Peris, 2017), la Escuela Bosque sigue un modelo educativo alternativo a la escuela tradicional en el que, en lugar de tener clases cerradas, los niños aprenden los contenidos curriculares en la naturaleza. Además, según Bruchner (2017b) el entorno natural ofrece las posibilidades idóneas para trabajar las competencias propias del currículo que necesitan para llevar una vida académica exitosa.

Por otro lado, para O'Brien (2009) este tipo de escuelas ayuda a los niños dándoles la oportunidad de alcanzar confianza y autoestima a través de experiencias en entornos naturales. Además, este entorno hace que se desarrollen juegos creativos e imaginativos y experiencias que harán que los niños desarrollen una actitud responsable ante los riesgos por medio de rutinas de seguridad y límites establecidos (Murray y O’Brien, 2005, citado en Smith, Dunhill y Scott, 2017).

El objetivo de este tipo de escuelas, según Odile Rodríguez de la Fuente (2015, Forminaya, 2015, p. 1):

Es preparar a las próximas generaciones de niños para abordar con éxito los nuevos desafíos de la sociedad. Que sean personas respetuosas con el medio en el que viven y conscientes de la necesidad de optimizar sus recursos, se sientan conectados con la tierra, y puedan potenciar al máximo sus aptitudes mediante el desarrollo de sus capacidades.

En definitiva: "la educación en la naturaleza es educación en, sobre y en la naturaleza" (Ford, 1986, p. 2). 


\section{La naturaleza como espacio de aprendizaje}

La principal característica de este tipo de escuelas es el establecimiento de la naturaleza como aula. Todas las actividades que se llevan a cabo son, precisamente, en el exterior. Los alumnos no están en una habitación sentados según el el binomio maestro-alumno, sino que realizarán actividades en el exterior, y será a través de estas actividades como se alcance el aprendizaje (Peris, 2017).

En definitiva, la naturaleza brinda numerosos recursos didácticos. Por ejemplo, es posible utilizar los diferentes materiales que se encuentran en la naturaleza (hojas, flores, palos, piedras...), para enseñar la grafía de las letras. Además, la forma de las piedras puede servir para explicar nociones matemáticas como el tamaño, al mismo tiempo que se enseña la cantidad de un número utilizando palos y figuras geométricas. La asociación In natura nos deja algunas ideas para incorporar la naturaleza como espacio y como recurso educativo.

\section{ideAs para trabajar la lecto-escritura}

\section{EN LA NATURALEZA}

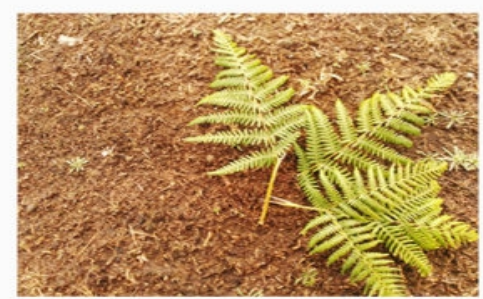

Identificación y Descripción

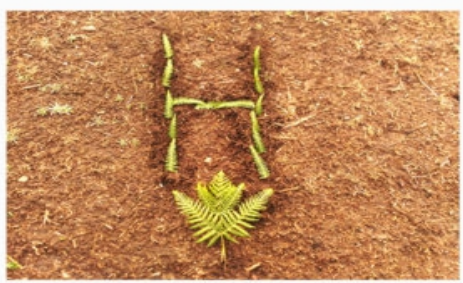

Asociar letra y elemento

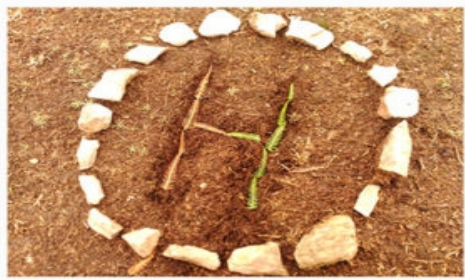

Trabajar con los colores y las simetrías

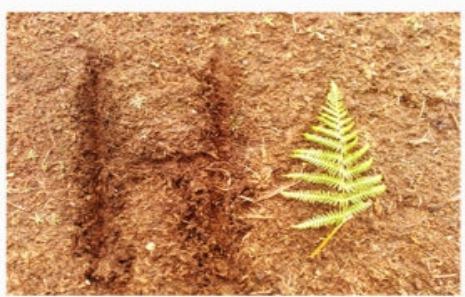

Iniciación al código escrito

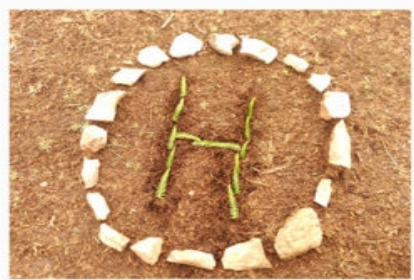

Crear un cuadro

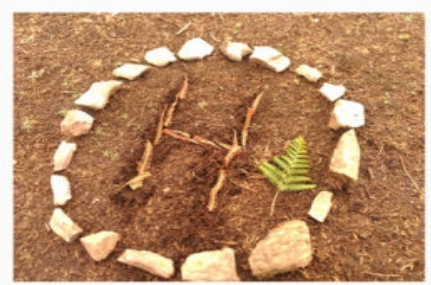

Crear "Ia historia del helecho"

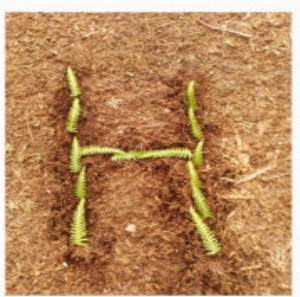

Rellenar la letra con el elemento (helecho)

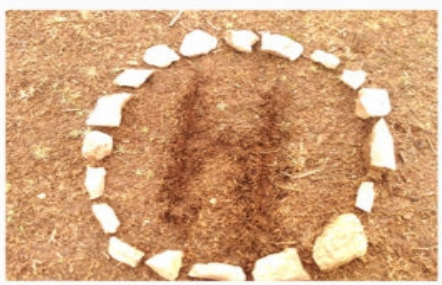

Trabajar la direccionalidad.

Decir otras palabras que comiencen por la misma letra

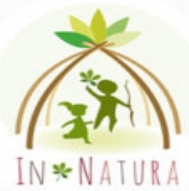

www.escuelainnatura.com info@escuelainnatura.com

Figura 1. Ideas para trabajar la lecto-escritura en la naturaleza. Fuente: https://escuelainnatura.com/matematicas-y-lecto-escritura-en-la-naturaleza/ 


\section{IDEAS PARA TRABAJAR LAS MATEMÁTICAS EN LA NATURALEZA}

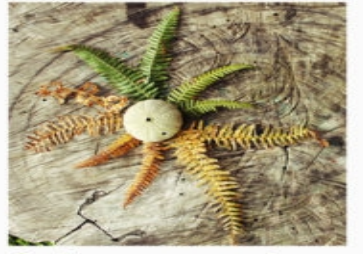

Ordenar por colores

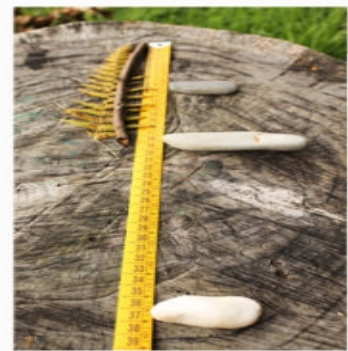

Registro de mediciones. Variedad en documentación

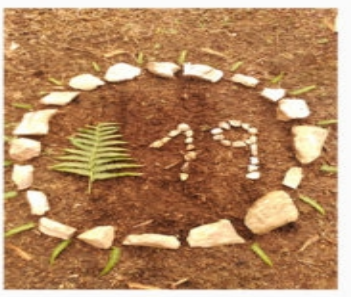

Conteo Juegos de Adición

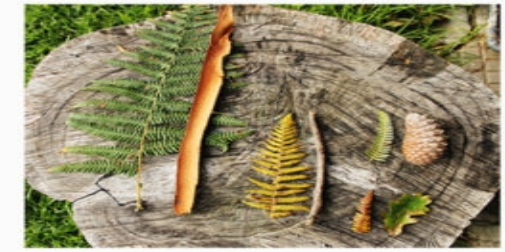

Encontrar elementos del mismo tamaño. Variedad de objetos
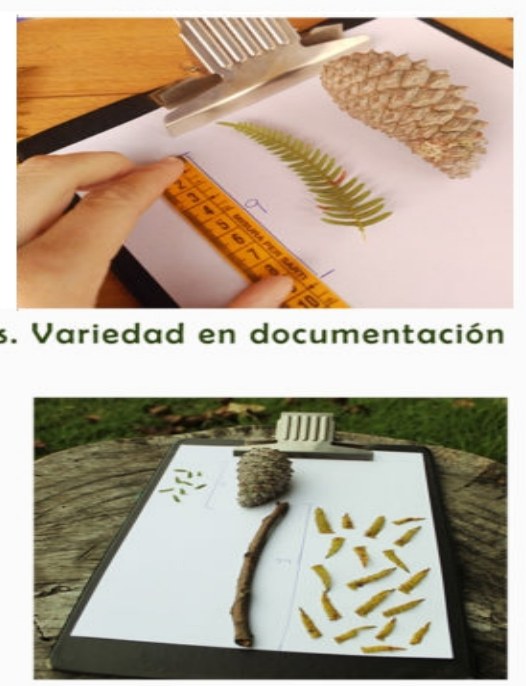

Equivalencias

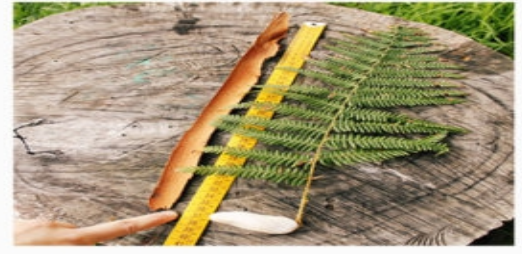

Tomar mediciones. Conceptos de longitud, anchura...

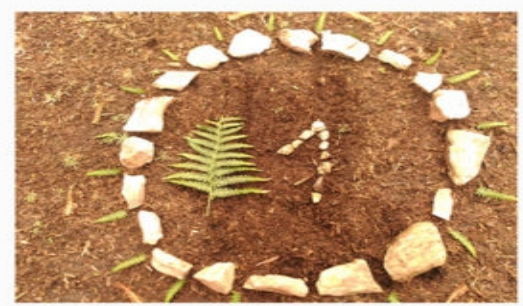

Pizarra natural.

Crear grafia del número de helechos

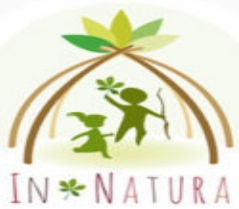

www.escuelainnatura.com info@escuelainnatura.com

Figura 2. Ideas para trabajar las matemáticas en la naturaleza. Fuente: https://escuelainnatura.com/matematicas-y-lecto-escritura-en-la-naturaleza/

Por añadidura, a través de la naturaleza se puede trabajar el concepto de tiempo pues, como apunta Tonda (2001, citado en Trigueros et al. 2013), la mejor forma de hacerlo es a través del medio ya que, si al introducir los fenómenos que ocurren en el medio, se nombra cuándo sucedió, se ayudará también a analizarlo. Si en una Escuela Bosque los niños observan día a día los árboles, las plantas e incluso las especies que los rodean, podrá entender, con ayuda del maestro, que las plantas no son las mismas en cada momento del año, que las especies que rondan van cambiando según las migraciones o que los árboles atraviesan momentos de cambio según la estación del año.

En otra instancia, a través de la naturaleza podrán entender mejor cómo es y cómo funciona el ciclo vital. Hay ocasiones en las que un animal es devorado por otro porque este lo necesitaba para sobrevivir y estos restos son, además, aprovechados como alimento para otros insectos. Así, la naturaleza forma un todo del que nosotros también formamos parte. 


\section{Día a día}

Como regla general, la jornada se organiza en torno a ciertos momentos concretos: un saludo matutino, senderos por los que los niños se desplazan y descubren la naturaleza de su entorno, el juego libre y las actividades programadas (GarcíaGonzález y Schenetti, 2019). Esta estructuración da seguridad y confianza a los más pequeños.

Como ejemplo de lo expuesto, en el Espacio Saltamontes, los niños llegan a las 8:30h, los recibe un acompañante en el edificio de referencia y se preparan para las condiciones meteorológicas de ese día. Hasta las 9:30h pueden seguir llegando niños y los padres que lo deseen pueden quedarse acompañando a sus hijos. A partir de esa hora, los padres se van retirando y comienza el momento del encuentro con el bosque. Tras esto, se realiza la subida al monte (figura 3). Sin embargo, no es una ruta como tal, sino que las niñas y los niños van descubriendo a su paso los detalles de la naturaleza. Una vez terminado el paseo, toman un tentempié y realizan juego libre. Sobre las 12:30h, todos vuelven al Espacio Saltamontes y comienza el momento del cuento mientras descansan tras la jornada. Finalmente, se disponen en la puerta a esperar a sus familias (Hueso, 2019).

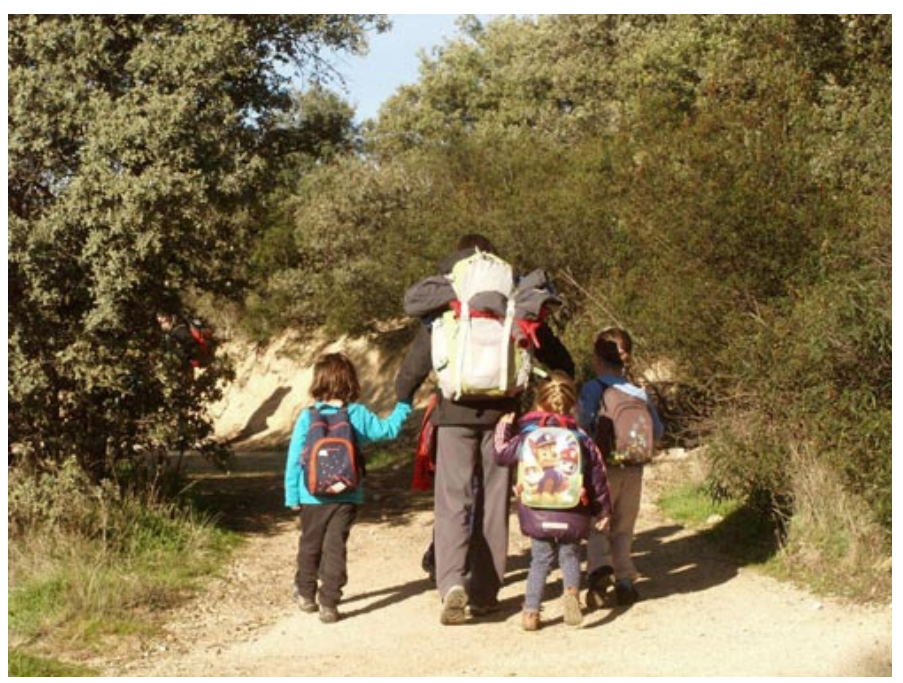

Figura 3. Alumnos de Grupo Saltamontes durante el paseo. Fuente:

https://ludus.org.es/es/blog/2019/10/un-dia-en-una-escuela-bosque\#.YFsuSEhKjpA

Por otra parte, en Bosquescuela Cerceda comienzan su rutina con una llegada tranquila en la cabaña. Después, tiene comienzo la clase dirigida, en la que se introducen contenidos propios del currículum. Para elegir el lugar donde se va a realizar la clase, el guía encargado de impartirla realiza un paseo de inspiración antes del inicio de la jornada. Tras la clase dirigida, tiene lugar el camino de aprendizaje, que es un paseo a un lugar específico que suele estar a 1000 metros de la cabaña. Cuando llegan, realizan el almuerzo y el juego libre (figura 4). Cuando ya han realizado el paseo de vuelta y se encuentran de nuevo en los alrededores, tiene lugar el relato del cuento. Tras comentarlo, vuelven a la cabaña a esperar a las familias o a que dé comienzo el comedor (Bruchner, 2017a). 


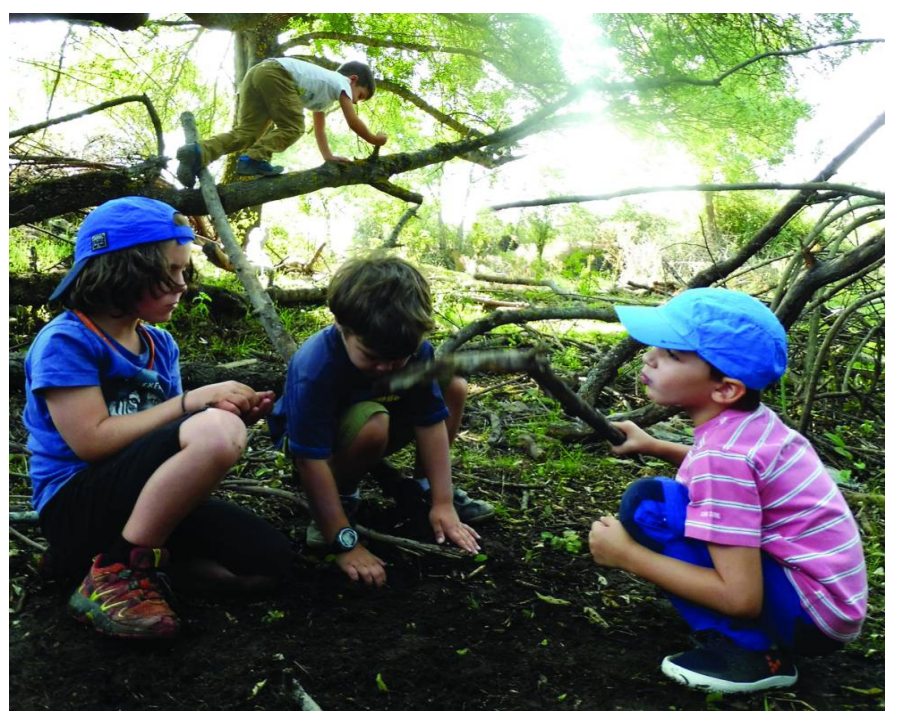

Figura 4. Varios alumnos de Bosquescuela en la Dehesa Boyal de Cerceda (Madrid). Fuente: https://elpais.com/economia/2018/02/07/actualidad/1517996397_579992.html

\section{Espacio}

A pesar de que no hay dos Escuelas Bosque iguales, sí podemos saber que hay características que comparten entre sí. Por ejemplo, todas deberían tener un edificio de apoyo en el que los niños puedan acudir en circunstancias climatológicas graves o, simplemente, para guardar ciertos materiales (García-González y Schenetti, 2019).

No obstante, eso no quiere decir que una Escuela Bosque sea un edificio que se sitúe en medio de la naturaleza, significa contar con la naturaleza como medio de aprendizaje. Por ello, el espacio en el que se sitúe la escuela debe ser amplio, con variedad de recursos naturales y posibilidad de manipularlos. Es decir, los alumnos deben poder excavar hoyos, escalar, etc. (Edna, 2021).

Para que una escuela pueda denominarse Escuela Bosque, debe tener instalaciones que mantengan las siguientes funciones: protección, orientación, descanso, comedor y almacenaje (Bruchner, 2017a). Durante los días que haga más frío o llueva intensamente, se debe mantener a los alumnos cerca de las instalaciones, que deben ser seguras para la óptima protección de los niños.

\section{Mezcla de edades}

En primer lugar, se debe saber que, según la Asociación de Educación en la Naturaleza (Edna, 2021), la ratio debe ser 1 guía por 8 alumnos. Otra de las características de las Escuelas Bosque que a su vez es compartida con las características de las escuelas alternativas, es la mezcla de edades. Según García (2016), en este tipo de escuelas no se considera un problema que haya niños con diferentes niveles en el mismo entorno de aprendizaje. De hecho, defiende que esta diversidad resulta muy beneficiosa. Si se tiene en cuenta que la educación persigue preparar a los niños para la sociedad actual, la enseñanza del trabajo con personas diferentes a ellos mismos les ayuda a enfrentarse a situaciones reales.

Los más pequeños pueden aprender de los mayores, siendo además estos su motivación para "hacerse grandes". Por otro lado, los mayores aprenden mucho mejor 
si tienen pequeños a los que "explicar" lo que ya saben y, además, mejoran su conducta al considerarse un ejemplo que seguir (García, 2016). Asimismo, estos alumnos forjan una relación en la que el mayor no se impone sobre el pequeño, sino que se crea una complicidad especial entre ellos. Este tipo de organizaciones resultan muy dispares de la realidad que se tiene en las aulas convencionales, marcadas por un currículum de referencia que marca qué debe saber un niño y cómo (Bustos, 2007).

En estas escuelas lo principal es que todos pueden aprender de todos, teniendo siempre en cuenta cuál es el desarrollo evolutivo de los niños, es decir, en qué momento se encuentran. Todo ello, pudiendo estar en contacto con una variedad de estímulos educativos (Boix y Bustos, 2014). Pues, ¿tenemos todos las mismas capacidades en todas las áreas? Evidentemente, no. Si bien podemos tener una habilidad matemática "pobre", podemos ser muy hábiles con el dibujo, por ejemplo. Esto tiene su base en la Teoría de las Inteligencias Múltiples de Gardner. Este consideró que todos los individuos tenemos diferentes tipos de inteligencias, y que el sistema educativo debe tener en cuenta esta teoría para tratar a los alumnos desde un modelo más justo (Gardner, 2005).

\section{Papel del docente}

Uno de los aspectos más importantes de las escuelas alternativas, que también comparten las Escuelas Bosque, es el papel del docente como guía. Esto significa la ruptura con la visión tradicional, lo que supone que sus intereses, motivaciones y ritmos de aprendizaje sean respetados. Se rehúye, además, del aprendizaje memorístico pues, se entiende que solamente se aprende aquello que el niño, motivado por sus intereses, experimenta a través de sus sentidos.

Siendo el niño el protagonista de su aprendizaje, el maestro guía al alumno hacia sí mismo. El maestro orienta al alumno hacia dónde van a caminar ese día, determina cuándo acaban los momentos de desayuno o juego libre, inspira a los alumnos con actividades de iniciación a la lectoescritura o a las matemáticas, etc.

Además, vela por la seguridad de los niños. En una Escuela Bosque, donde el entorno de aprendizaje es la naturaleza, el papel del docente como guardián de la seguridad de los niños, es principal. El maestro deberá comprobar la peligrosidad de los árboles antes de que los niños comiencen a trepar y controlar que todos los niños lleven la ropa adecuada para salir al exterior.

Partiendo de la experiencia de Marga Keller (maestra de una Bosque Escuela en Suiza), "en las escuelas bosque se producen menos accidentes que en las escuelas ordinarias" (Fahey, 2015, p. 17).

\section{Evaluación}

Como ya se ha mencionado anteriormente, estas escuelas están enmarcadas en lo que hoy día se conoce como "escuelas alternativas". Una de las principales señas de identidad de estas escuelas es la evaluación sin exámenes. Según esta postura, para poder evaluar al niño es necesario entenderlo para poder llegar a lo que realmente está ocurriendo en su interior. Rechazan, así, la calificación como un método de evaluación. 
En contra de esto, utilizan la observación para poder hacer un seguimiento del proceso educativo. Sin embargo, esto no debe entenderse como una mera ojeada, sino como una recogida de información en primer lugar, y análisis exhaustivo final. Según un estudio realizado por Benítez (2017) en el que se analizaba el sistema de evaluación de seis Escuelas Bosque, se dedujo que el método más utilizado era "la observación y anotación de las capacidades o habilidades desarrolladas a lo largo del trimestre, las cuales se comentan con las familias al finalizar cada trimestre" (p. 44).

Por lo tanto, la evaluación de estas escuelas no se limita a una calificación, sino a una consecución de logros y capacidades de manera progresiva y respetando el ritmo de los niños. Bulit (2020) analizó dónde se comenzó a hablar sobre calificaciones en las escuelas, y dedujo que fue en las Universidades. Poco a poco, este sistema se fue extendiendo hasta llegar a las aulas de los más pequeños, sin tener en cuenta el efecto que supondría el "premiar" los aciertos y "castigar" los errores en sus mentes. Además, apunta Bulit (2020) que según artículos de New York Times las calificaciones elevadas en la escuela no suponen siempre un éxito profesional. Entonces, ¿tiene sentido seguir sobre calificando a los niños?

\section{Ventajas e inconvenientes}

\section{Naturaleza y aprendizaje}

Una de las ventajas que implica la naturaleza en el aprendizaje es su función educativa. El juego en la naturaleza tiene un gran potencial para el aprendizaje y para el desarrollo psicológico del niño. Los niños de educación infantil se hallan, según Piaget, en la etapa preoperacional del pensamiento. Esta se caracteriza por el surgimiento del razonamiento, el incremento del uso de conceptos y, en especial, por el aumento del uso del pensamiento simbólico (Feldman, 2007). Según este, el niño utiliza objetos para recrear acciones propias de su vida cotidiana, como llamar por teléfono usando un plátano, para fingir representaciones fantásticas y, en definitiva, para vivir sus propias aventuras. Este tipo de juego favorece, según Tomás y Almenara (2007), el desarrollo del lenguaje, las habilidades sociales y cognoscitivas y, además, la creatividad y la imaginación.

En cuanto a las características de los recursos que los niños pueden encontrar a la hora de jugar al aire libre en la naturaleza, sus posibilidades son inmensamente amplias. Un palo, una hoja, una piedra, una flor, etc., pueden ser infinitas cosas. Siguiendo a Louv (2005, p. 23), "la naturaleza sirve como una pizarra en blanco sobre la cual niños y niñas dibujan y reinterpretan las fantasías de la cultura”.

Todo aquello que produce curiosidad, que provoca misterio y que despierta los sentidos, es lo que llega a lo más profundo del niño y de lo que no se desprenderá jamás. Los niños rebosan de esa curiosidad, como apunta Carson (1956) en lo que denomina el sentido del asombro. Carson defiende que es esa mente intuitiva y sensorial lo que facilita que el niño aprenda a una velocidad vertiginosa y, por tanto, la naturaleza es la mayor fuente de sonidos y sensaciones que un niño puede encontrar.

Recuperando las ideas que se han heredado de la ILE, se observa que el niño conoce, aprende y descubre el mundo a través de los sentidos, y no es sino la naturaleza, el lugar idóneo donde encontrar esos estímulos. 
De esta manera, partiendo del pilar de la neuroeducación que da nombre a la obra de Francisco Mora: "solo se puede aprender aquello que se ama", se advierte que el niño necesita disfrutar, soñar, gritar, en definitiva, necesita amar para aprender. La naturaleza, además de propiciar el ambiente idóneo para que se produzcan estas emociones, permite la expresión libre del movimiento, ingrediente base para que se produzca el aprendizaje. El movimiento unido con las experiencias directas y sensitivas provoca la activación de los sistemas sensoriales, motores y de diferentes partes del cerebro. Además, a través del movimiento se produce un aumento de oxigenación en el cerebro que permite que se construyan habilidades cognitivas y capacidades mentales, sociales y emocionales (Campos, 2010).

En última instancia, cabe resaltar los beneficios en el nivel cognitivo que se han demostrado en niños de Educación Primaria según su escolarización en la etapa de Educación Infantil. En 2002 se llevó a cabo un estudio en la Universidad de Heidelberg en el cual se pedía a los profesores que evaluaran a los alumnos según ciertas competencias (figura 5). A continuación, se compararon los resultados de los alumnos que habían ido a una escuela al aire libre con los que acudieron a una escuela ordinaria.

\section{Competencias adquiridas por el alumnado de primaria según su escolarización en infantil}

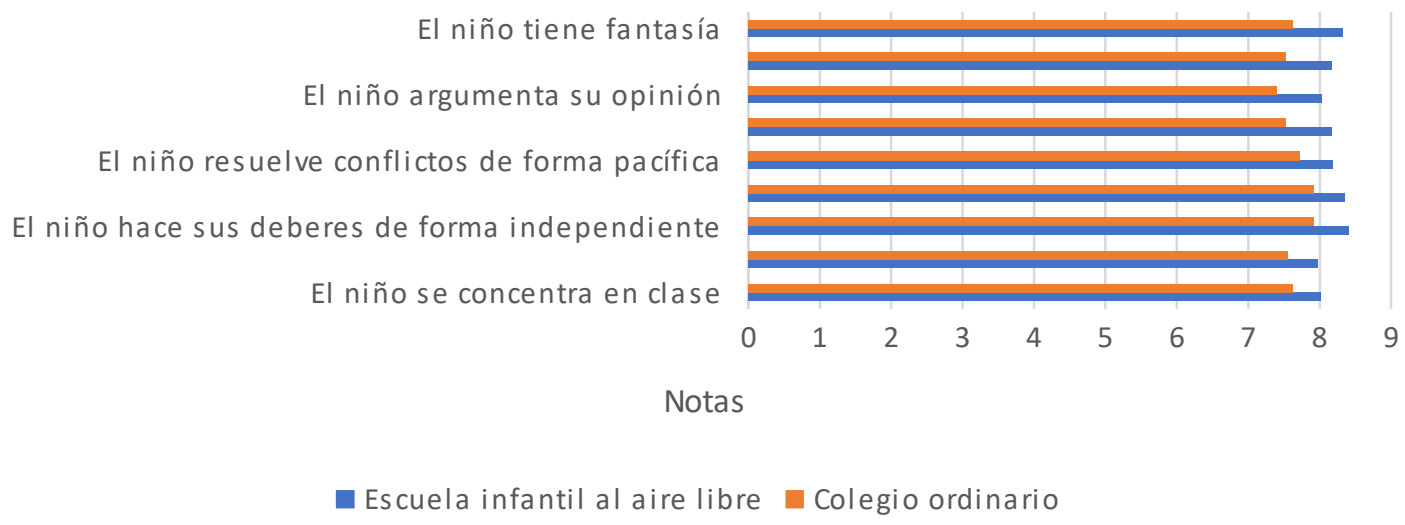

Figura 5. Representación gráfica de los resultados del estudio de la Universidad de Heidelberg (Bruchner, 2012).

Los resultados evidencian que los alumnos que acudieron a una escuela al aire libre siguen mejor las clases, mantienen mejor la atención, son más independientes, resuelven los conflictos con más calma, tienen una mejor argumentación y más fantasía (Bruchner, 2012).

\section{Naturaleza y salud}

Como ya se ha comentado al inicio de este trabajo, la sociedad está atravesando una crisis de identidad natural promovida por el exceso de urbanidad y los cambios de forma de vida. Un estudio realizado en Reino Unido señalaba que los niños a partir de seis años criados en ambientes urbanos podían identificar más Pokemon que plantas o animales locales reales (Balmford et al. 2002). 
En consecuencia, los niños se han visto afectados por estos cambios. Partiendo de la teoría de Louv (2005), aquellas "enfermedades" modernas como el estrés, la depresión, el trastorno por déficit de atención e hiperactividad (en adelante, TDAH), podrían ser causadas, precisamente, por esta falta de contacto con el mundo natural. Según este autor, se podría tratar de un nuevo término acuñado por él mismo como trastorno por déficit de naturaleza.

Con esta afirmación, no pretende llegar hasta un diagnóstico clínico, sino plantear una hipótesis que ayude a comprender a los niños del siglo XXI. De hecho, según sus investigaciones, gracias al efecto calmante y restaurador de la naturaleza, esta podría resultar muy útil como terapia (alternativa o combinada con otras propias del TDAH), para los niños que están diagnosticados de este trastorno. Así mismo, puede ser beneficioso para niños con espectro autista, aquellos con dificultades emocionales y de comportamiento y con problemas de aprendizaje (Kahn, 1999; Forestry Commision Scotland, 2005; O’Brien, 2005; O’Brien y Murray, 2006; Borradaile, 2006 citado en O’Brien, 2009).

Con respecto a la obesidad infantil, considerada como la epidemia del siglo XXI, Liu, Wilsom y Qi Ying (2007, citado en Collado y Corraliza, 2016) realizaron un estudio sobre la relación entre la cantidad de vegetación que rodea los lugares de residencia de los niños y el grado de sobrepeso infantil. Gracias a esto, se conoce que una mayor cantidad de vegetación en la zona de residencia se asocia con un menor riesgo de sobrepeso infantil. Además, Ozdemir y Yilmar (2008, citado en Collado y Corraliza, 2016) relacionaron las características de los patios de los colegios (espacios abiertos, árboles y agua) con el índice de masa corporal de los niños, así como de sus sentimientos y bienestar durante el recreo.

Según Cathy Bache, directora de la Escuela Bosque Jardín Secreto, las posibilidades que brinda una escuela bosque a los niños permiten que los niños tengan gran cantidad y diversidad de experiencias que favorecen la libertad de movimientos, los cuales contribuyen a aumentar su fortaleza física y emocional, su sentido de la independencia y eficacia personal y la confianza en sus capacidades (Freire, 2011).

\section{Comportamientos proambientales}

Siguiendo con los beneficios que puede conllevar el contacto directo y continuado de la naturaleza en los niños de educación infantil, se debe resaltar los beneficios a nivel medioambiental.

Teniendo en cuenta que las experiencias que los niños tienen en las edades más tempranas serán aquellas que más influyan en su desarrollo psicológico y emocional posterior, en este tipo de escuelas donde los niños sienten en la naturaleza libertad y calma, los niños crearán un contacto positivo con la naturaleza que les durará toda la vida. Este contacto positivo dará pie a lo que, gracias a Kals et al. (1999, citado en Collado y Corraliza, 2016), se conoce como afinidad emocional hacia la naturaleza (en adelante, EAN), cuyo significado se puede desglosar en cuatro conceptos: "el amor a la naturaleza, la sensación de libertad en la naturaleza, el sentimiento de seguridad en la naturaleza y los sentimientos de unidad con la naturaleza" (Collado y Corraliza, 2016, p. 123).

Turtle, Convery y Convery (2015, citado en Smith, Dunhill y Scott, 2017), expusieron que había una diferencia estadísticamente significativa en la actitud proambiental, es 
decir, en la conciencia ecológica y preocupación por el medio ambiente y sus componentes, entre los niños que habían asistido a una Escuela Bosque y aquellos que no. Además, los niños que van a Escuelas Bosque se vuelven más conscientes, se interesan y desean aprender más sobre el ambiente natural. Estos niños pueden, incluso, desarrollar el conocimiento para nombrar animales y árboles y empezar a comprender conceptos ecológicos (Smith, Dunhill y Scott, 2017).

Además, Kals et al. (1999, citado en Bethelmy, 2012) sugieren que esta atracción hacia la naturaleza tiene relación con lo que se conoce como biofilia. Este concepto, según Wilson (1984, citado en O’Brien y Murray, 2007), sugiere que tenemos una afinidad innata con la naturaleza que hace que nuestras acciones siempre vayan encaminadas hacia ella. Esta explica que los seres humanos tiendan a cuidar la naturaleza porque se sienten parte de ella ya que, al fin y al cabo, son seres naturales. Siguiendo a Collado y Corraliza (2016), si ese instinto natural se ve desarrollado desde pequeños, crecerán adultos con un mayor sentimiento ecológico y de cuidado al medio ambiente.

Por otro lado, esta forma de enseñanza entraña diversos inconvenientes. Actualmente se advierte una explosión de nuevas propuestas e ideas de escuelas que priman el interés de los alumnos y que opinan que son estos, impulsados por su motivación, los que deben construir su conocimiento, y que la escuela es un lugar en el que deben aprender a aprender. Como ya se ha mencionado anteriormente, la Escuela Bosque se sitúa dentro de esta corriente que se conoce como educación alternativa. Sin embargo, en contra de estas se han posicionado muchos autores.

En primer lugar, Moreno (2006) alega en Panfleto antipedagógico que, en contra de lo que defienden las metodologías no convencionales, se debe fomentar la memoria y el uso de los libros. Según este, sin los libros los alumnos no pueden mejorar su capacidad memorística que es, según el autor, la base de la madurez humana.

Además, hay quienes opinan que en estas escuelas se les pone demasiado fácil el nivel de aprendizaje y que la escuela no es un circo donde estos van a divertirse. En palabras de Geli (2007 citado en García, 2016, p. 201):

Es evidente que la falta de educación es ahora superior a la hace unos años. ¿Qué ha pasado? Pues demasiados años de Rosa Sensat, plastilina y todo eso. Y de unos padres que crecieron confundiendo la autoridad con el fascismo. Educar es reprimir.

Teniendo esta postura en cuenta, es posible el acercamiento a uno de los principales inconvenientes que tiene este tipo de escuelas: la posterior inclusión de estos niños a la sociedad. García (2016) opina que suele ser una situación dura, ya que pasar de tener total libertad de movimiento a quedarse sentado en un pupitre toda la mañana, el ruido de las aulas, los gritos de los niños, etc., puede suponer un cambio muy difícil.

Además, también hay motivos por los que, en escuelas convencionales, no se utiliza la naturaleza como un espacio más de aprendizaje. Siguiendo a Yildirim y Özyilmaz (2017), a pesar de que los profesores tienen una actitud proactiva a realizar actividades en la naturaleza, tienen miedo de que los niños enfermen y de la negativa de los padres. También los espacios verdes son insuficientes y no cuentan con los recursos necesarios.

Por otro lado, se hace referencia a que esta escuela no es apropiada para todos los niños. Si bien se ha demostrado que para los niños con TDAH este modelo puede 
llegar a ser muy beneficioso, no ocurre lo mismo con los niños con un grado de autismo grave, pues al tener que tomar sus propias decisiones todo el tiempo, puede que no sepan desenvolverse (Gray, 2016 citado en García, 2016).

De otra parte, Peris (2017) sugiere que hay otros factores por los que no se lleva a cabo una educación en la naturaleza como la falta de recursos económicos de los colegios, la actitud sobreprotectora de los padres, la escasa formación en educación en la naturaleza de los profesores y las obligaciones que tienen los colegios por cumplir unos objetivos y establecer exámenes.

Asimismo, se advierte otro gran inconveniente: el precio de estas escuelas. La mayoría de estos proyectos suelen estar privados de subvenciones, por lo que la cuota mensual suele ir de $100 €$ a $400 €$ al mes (García, 2016, p. 156). Por tanto, esto provoca que no todas las familias puedan permitirse este gasto y que, en muchas ocasiones, los maestros de estas escuelas vivan en precariedad donde lo que les mueve es más la ilusión que la motivación salarial (García, 2016).

Otra de las sombras que tiene este tipo de metodología es la mercantilización. Sanchidrián (2020) ha analizado la lista de escuelas recogidas en la plataforma Ludus y se ha encontrado con Escuelas Bosque que, además, utilizan materiales Montessori; otras se anuncian como un espacio de juego en la naturaleza en el que realizan talleres artísticos, etc. Así, apunta que a pesar de que se han realizado estudios que demuestran la efectividad de estas metodologías, los resultados no pueden ser concluyentes, puesto que hay centros que admiten que hacen una selección de sus alumnos.

\section{Conclusión}

La Escuela Bosque se enmarca como un modelo educativo que está emergiendo en todo el mundo, especialmente en nuestro país. Gracias a este breve análisis de sus principales características se demuestra que la naturaleza sí que puede tener un lugar de importante relevancia en el proceso de enseñanza-aprendizaje.

A través de ella, se puede aprender matemáticas, lenguaje, ciencias y, no solo eso, sino que además el alumnado se puede beneficiar de los grandes tesoros de la naturaleza: un mejor estado de salud (evitando la obesidad o reduciendo la hiperactividad), un entorno libre de enfermedades víricas y adquisición de comportamientos proambientales que serán útiles tanto a nivel personal como para la sociedad.

Asimismo, el modo de actuación de estas escuelas resulta todavía extraño en nuestra sociedad. La mezcla de edades dentro de una misma aula, el papel del docente como guía en el aprendizaje, o la evaluación "sin exámenes" son aspectos de este tipo de escuelas que están todavía lejos de las escuelas tradicionales. Sin embargo, se puede extraer la esencia de ellas para llevarla a las escuelas convencionales: la naturaleza como espacio educativo. Por ello, difundir esta información resulta fundamental para que haya un cambio real en la sociedad y, en concreto, en la educación.

Como hemos comentado, uno de los inconvenientes con los que nos encontramos a la hora de incorporar en las escuelas convencionales la naturaleza, es la falta de formación del profesorado en este ámbito. Con estudios como este, se pretende dar solución a esa falta de conocimiento sobre los cambios educativos que se están llevando a cabo. 
Por otro lado, resulta esencial tomar esta nueva forma de enseñanza que está emergiendo como una posible respuesta a la situación de pandemia que estamos viviendo. Cada vez son más los centros educativos que, partiendo de los ejemplos de las Escuelas Bosque, salen a los parques y las zonas naturales que tienen cerca, para estar menos tiempo en los lugares cerrados. Como apunta Lucas (2020), el entorno natural tiene mucha más seguridad ya que los materiales naturales no necesitan desinfección, hay más posibilidad para mantener la distancia de seguridad y, al pasar casi el $80 \%$ del tiempo en la naturaleza, el aire se mantiene renovado.

Esto es por lo que luchan desde hace meses la asociación SEO Bird Life (que cuenta con proyectos como Escuelas Naturales por el cambio, o Aulas Libera) (SEO BirdLife, 2021), o la asociación Edna (2020, p. 2), que elaboró un manifiesto por la infancia con la defensa de que "la educación en la naturaleza puede brindar una solución inmediata a casi todos los problemas a la vez que aborda la causa última de la pandemia". Este manifiesto ha sido firmado y respaldado por casi setenta asociaciones con una causa común: unir la naturaleza y la infancia para mejorar la calidad de vida de las personas.

Con todo, como los ya citados Colegio Félix Rodríguez de la Fuente de Murcia (Ribelles, 2021), la escuela Turó del Cargol en Barcelona (Ribalaygue, 2020) o la escuela Ports de Xàbia (Morales, 2020), cada vez son más las escuelas que, partiendo de las ventajas que nos muestran las Escuelas Bosque, incorporan la naturaleza en su día a día. Por tanto, como defiende García (2016): "Otra educación ya es posible".

\section{Referencias}

Acuña, S., Díaz, J., \& Ramírez, K. (2018). Medio ambiente y salud: factores ambientales que influyen en las condiciones de vida. Revista Vinculando, 1-9. http://vinculando.org/salud/medio-ambiente-y-salud-factores-ambientalesque-influyen-en-las-condiciones-de-vida.html

Aguado, C. (2018). Rosa Sensat y la primera Bosque Escuela de España, 1914. Inedit Magazine. http://www.ineditweb.es/rosa-sensat-y-la-primera-bosque-escuelade-espana-1914-art600

Balmford, A., Clegg, L., Coulson, T., \& Taylor, J. (2002). Why conservationists should heed Pokémon. Science, 295(5564), 2367. http://dx.doi.org/10.1126/science.295.5564.2367b.

Benítez, A. (2017). ¿Qué es la educación alternativa y qué características tiene? Un estudio comparativo en la provincia de Sevilla. Trabajo final de grado, Grado en Educación Infantil,. Sevilla: Universidad de Sevilla. https://idus.us.es/handle/11441/63062

Bethelmy, I. (2012). Experiencia de lo sublime en la vinculación emocional con la naturaleza. Una experiencia de la orientación proambiental. Tesis doctoral. Madrid: Universidad autónoma de Madrid. https://repositorio.uam.es/bitstream/handle/10486/662506/bethelmy_rinco n_lisbeth_carolina.pdf?sequence $=1$

Boix, R., \& Bustos, A. (2014). La enseñanza en las aulas multigrado: Una aproximación a las actividades escolares y los recursos didácticos desde la perspectiva del 
profesorado. Revista Iberoamericana de evaluación educativa, 7(3), 29-43. https://revistas.uam.es/riee/article/view/3100

Bruchner, P. (2012). Escuelas infantiles al aire libre. Cuadernos de pedagogía, (420), 26-29. https://bosquescuela.com/wp-content/uploads/2012/01/Art \%C3\%ADculo_Escuelas-infantiles-al-aire-libre_Cuadernos-de-Pedagog $\%$ C3\%ADa1.pdf

Bruchner, P. (2017a). Bosquescuela. Guía para la educación al aire libre. Valencia: Ediciones Rodeno.

Bruchner, P. (2017b). Taller de iniciación en metodología Bosquescuela. (Material no publicado).

Bulit, D. (9 de septiembre de 2020). ¿Cómo evaluar sin_calificar? Así o hacen las escuelas experimentales, Waldorf y Montessori desde siempre. Alteredu. https://alteredu.com.ar/2020/09/09/como-evaluar-sin-calificar-asi-lo-hacenlas-escuelas-experimentales-waldorf-y-montessori-desde-siempre/

Bustos, A. (2007). Enseñar en la escuela rural aprendiendo a hacerlo. Evolución de la identidad profesional en las aulas multigrado. Profesorado. Revista de currículum y formación de profesorado, 11(3) 3-26. . https://recyt.fecyt.es/index.php/profesorado/article/view/42614

Campos, A. (2010). Neuroeducación: Uniendo las neurociencias y la educación en la búsqueda del desarrollo humano. La educ@ción, (143), 1-14. http://kdoce.cl/wp-content/uploads/2017/10/DOC1-neuroeducacion.pdf

Carson, R. (1956). El sentido del asombro. Madrid: Ediciones Encuentro.

CIFREP. (2021). Children's International Foundation for Research Education and Peace. https://cifrep.org/

Collado, S., \& Corraliza, J. (2016). Conciencia ecológica y bienestar en la infancia. Efectos de la relación con la naturaleza. Madrid: Editorial CCS.

Edna (2020). Covid-19. Manifiesto por la infancia. Federación Edna de Educación en la naturaleza.

https://asociacionedna.files.wordpress.com/2020/05/manifiesto_20_05_151.pdf

Edna (2021). Federación Edna de Educación en la naturaleza. https://asociacionedna.wordpress.com/

Fahey, D. (2015). Forest Schools and the Benefits of Unstructured Outdoor Play. Clearing magazine, 16-18. Compendium. http://clearingmagazine.org/archives/8520

Feldman, R. (2007). Desarrollo psicológico a través de la vida. México D.F., México: Pearson.

Ford, P. (1986). Outdoor Education: Definition and Philosophy. ERIC Clearinghouse on Rural Education and Small Schools. https://eric.ed.gov/?id=ED267941

Forestry Commission Scotland (2005). Public Opinion of Forestry 2005: Scotland. Results of the 2005 Scottish survey of Public Opinion of Forestry, carried out on behalf of the Forestry Commission. Edinburgh, U.K.: Forestry Commission. 
https://www.forestresearch.gov.uk/documents/3691/SCOTTISHPUBLICOPINI ONSURVEY2005.pdf

Forminaya, C. (15 de enero de 2015). Inauguran en España la primera bosque escuela alemana. Diario ABC. https://www.abc.es/familia-educacion/20140216/abciescuelas-aire-libre-201402141136.html

Freire, H. (2011). Educar en verde. Ideas para acercar a niños y niñas a la naturaleza. Barcelona: Graó.

García-González, E., \& Schenetti, M., (2019) Las escuelas al aire libre como contexto para el aprendizaje de las ciencias en infantil. El caso de la Scuola nel BoscoVilla Ghigi. Revista Eureka sobre Enseñanza y Divulgación de las Ciencias 16(2), 2204. https://revistas.uca.es/index.php/eureka/article/view/4621

García, A. (2016). Otra educación ya es posible. Una introducción a las pedagogías alternativas. Albuixech: Litera.

Gardner, H. (2005). Inteligencias múltiples. Barcelona: Paidós. http://www.materialestic.es/transicion/apuntes/Gardner,Howardinteligencias.multiples,la.teoria.en.la.practica(intro).pdf

Grooten, M. y Almond, R. (coords). (2018). Informe Planeta Vivo - 2018: Apuntando más alto. Gland, Suiza: http://awsassets.wwf.es/downloads/informe_planeta_vivo_2018.pdf? _ga $=2.261371149 .1451282619 .1547921511-562390383.1547921511$

Hueso, K. (2012). Mejores personas para un planeta mejor: El proyecto pedagógico al aire libre "Saltamontes", pionero en España. Congreso Nacional del Medio Ambiente.

http://www.conama2012.conama.org/conama10/download/files/conama11/ CT\%202010/1896699911.pdf

Hueso, K. (8 de octubre de 2019). Un día en una escuela en la naturaleza. Ludus. Recuperado de https://ludus.org.es/es/blog/2019/10/un-dia-en-una-escuelabosque\#.YM7uXC_WfpA

Kahn, E. (1999). A critique of nondirectivity in the person-centered approach. Journal of Humanistic Psychology, 39(4), 94-110. https://doi.org/10.1177/0022167899394006

Lahoz, P. (1991). El modelo froebeliano de espacio-escuela. Su introducción en España. Historia de la educación, 10, 107-133. Recuperado de http://revistas.usal.es/index.php/0212-0267/article/view/6915

Lahoz, P. (1991). El modelo froebeliano de espacio-escuela. Su introducción en España. Historia de la educación, (10), 107-133. http://revistas.usal.es/index.php/0212-0267/article/view/6915

Louv, R. (2005). Los últimos niños en el bosque: Salvemos a nuestros hijos del transtorno por deficit de naturaleza. Madrid: Capitán Swing.

Lucas, B. (15 de noviembre de 2020). Cuando el maestro es el bosque o la playa. El País. $\quad$ https://elpais.com/educacion/2020-11-14/cuando-el-maestro-es-elbosque-o-la-playa.html 
Ludus. (23 2021). Listado de proyectos de pedagogías activas y alternativas. https://ludus.org.es/es

Morales, E. (6 de septiembre de 2020). Un cole de Xàbia dará las clases al aire libre. Cadena

Ser. https://cadenaser.com/emisora/2020/09/06/radio_valencia/1599385629_48 0707.html

Moreno, R. (2006). Panfleto antipedagógico. Barcelona: El Lector Universal. https://www.cs.upc.edu/ conrado/docencia/panfleto-antipedagogico.pdf

Narváez, E. (2006). Una mirada a la Escuela Nueva. Educere, 10(35), 626-636. Recuperado de https://www.redalyc.org/articulo.oa?id=35603508

O’Brien, L. (2009). Learning outdoors: the Forest School approach. Education 3-13, International Journal of Primary, Elementary and Early Years Education, 37(1), 45-60. Recuperado de http://dx.doi.org/10.1080/03004270802291798

O’Brien, L., \& Murray, R. (2007). Forest School and its impacts on young children: Case studies in Britain. Urban Forestry \& Urban Greening, 6(4), 249-265. https://www.sciencedirect.com/science/article/pii/S1618866707000301

Peris, L. (2017). Outdoor education. Una forma de aprendizaje significativo. Sevilla: Punto Rojo Libros.

Ribalaygue, J. (11 de noviembre de 2020). El coronavirus estimula las clases al aire libre en escuelas de Barcelona. El Mundo. https://www.elmundo.es/cataluna/2020/11/11/5fabc1f1fc6c8331768b45e0. html

Ribelles, E. (23 de marzo de 2021). El colegio de los Nietos imparte clase a cien niños junto al Mar Menor. Periódico La Verdad. https://www.laverdad.es/murcia/cartagena/colegio-nietos-imparte20210323001823-ntvo.html

Ruiz, A. (2017). La estética ambiental en el bienestar de la comunidad escolar. Las Escuelas Bosque como referente. Trabajo Fin Grado en Educación Primaria. Universidad de Valladolid. http://uvadoc.uva.es/bitstream/10324/29556/1/TFG-0-1226.pdf

Sanchidrián, C. (2020). El método Montessori en la educación infantil española: luces y sombras. Historia de la Educación, (39), 313-335. https://doi.org/10.14201/hedu202039313335

Sanchidrián, C., \& Ruiz, J. (Coords.). (2010). Historia y perspectiva actual de la educación infantil. Barcelona: Graó.

SEO Birdlife. (8 de septiembre de 2021). Reclamamos mayor protagonismo de la naturaleza en las aulas y clases al aire libre Sociedad Española de Ornitología. https://seo.org/2020/09/08/en-la-vuelta-al-cole-reclamamos-mayorprotagonismo-de-la-naturaleza-en-las-aulas-y-clases-al-aire-libre/

Smith, M., Dunhill, A. y Scott, G. (2017). Fostering children's relationship with nature: exploring the potential of Forest School. Education 3-13, International Journal of Primary, Elementary and Early Years Education, 46(5), 525-534. http://dx.doi.org/10.1080/03004279.2017.1298644 
Tomás, J., \& Almenara, J. (2007). Máster en Paidopsiquiatría. Barcelona: Universidat Autònoma de

Barcelona. http://www.paidopsiquiatria.cat/archivos/teorias_desarrollo_cognitivo_0709_m1.pdf

Trigueros, F. J., Arias, L., Miralles, P., Molina, S., \& Rodríguez, R. A. (2013). Didáctica de las Ciencias Sociales en los cursos de grado de educación infantil. Murcia: Diego Marín.

Yıldırım, G., \& Özyilmaz, G. (2017). The effect of outdoor learning activities on the development of preschool children. South African Journal of Education, 37(2). http://dx.doi.org/10.15700/saje.v37n2a1378 